\title{
CAPACIDADE DE MANUTENÇÃO POSTURAL EM DIFERENTES ATIVIDADES FUNCIONAIS DE IDOSOS HIPERTENSOS E NÃO HIPERTENSOS
}

\author{
Posture maintenance capacity in different functional activities of hypertensive and non- \\ hypertensive elderly
}

\section{Capacidad del mantenimiento de la postura de ancianos hipertensos y no hipertensos en distintas actividades funcionales}

\author{
Jefferson Carlos Araujo Silva \\ Hospital Universitário Presidente Dutra - Universidade Federal do Maranhão - HU UFMA - São Luís (MA) - Brasil \\ Fuad Ahmad Hazime \\ Universidade Federal do Piauí - UFPI - Parnaíba (PI) - Brasil
}

Gaussianne de Oliveira Campelo

Hospital Universitário Presidente Dutra - Universidade Federal do Maranhão - HU UFMA - São Luís (MA) - Brasil

Luan Nascimento da Silva

Hospital Escola da Universidade Federal de Pelotas - HU UFPEL - Pelotas (RS) - Brasil

Mara Dayanne Alves Ribeiro

Universidade Federal do Ceará - UFC - Sobral (CE) - Brasil

Sabrynna Brito Oliveira

Universidade Federal de Minas Gerais - UFMG - Belo Horizonte (MG) - Brasil

\section{RESUMO}

Objetivo: Comparar a capacidade de manutenção postural estática e dinâmica em atividades funcionais entre idosos hipertensos e não hipertensos. Métodos: Participaram deste estudo indivíduos com idade igual ou superior a 60 anos, provenientes de uma Unidade Básica de Saúde (UBS) em um município de pequeno porte no norte do estado do Piaú. A capacidade de manutenção postural foi medida através de três testes. A mobilidade funcional foi ponderada através do teste Timed Up and Go (TUG); o equilíbrio estático, por meio do Teste de Alcance Funcional (TAF); e a força muscular (FM) dos membros inferiores, pelo teste Short Physical Performance Battery (SPPB). Os idosos foram divididos em grupo HAS (hipertensos) e grupo controle (não hipertensos). Os dados foram analisados através do Software Graph Pad Prism, utilizando o teste $t$ student não pareado para identificar diferenças entre os grupos. Resultados: Dos 88 voluntários, 43 pertencem ao grupo de hipertensos e 45 ao grupo controle. Para o TAF, o grupo HAS teve média da mensuração do alcance funcional de $15( \pm 5,60)$ centímetros, enquanto o grupo controle teve média de $17( \pm 5,88)$ centímetros. No teste SPPB, a média da FM para grupo HAS foi de $17,67( \pm 6,46)$ segundos e, para o grupo controle, foi de 17,01 $( \pm 3,53)$ segundos. A média do TUG para o grupo HAS foi $14,03( \pm 2,95)$ segundos e, para o grupo controle, foi $12,41( \pm 3,90)$ segundos. Houve associação entre grupos HAS e controle para os testes TAF $(p=0,03)$ e SPPB (p=0,02). Conclusão: A hipertensão arterial sistêmica parece interferir na manutenção postural estática em idosos.

Descritores: Hipertensão; Idoso; Equilíbrio Postural.

\section{ABSTRACT}

Objective: To compare static and dynamic posture maintenance capacity in functional activities between hypertensive and non-hypertensive elderly individuals. Methods: This study was held with participation of individuals aged 60 years or older, coming from a Basic Health Unit (BHU) in a small municipality in the north of the Piaui State. The posture maintenance capacity was assessed through the Timed Up and Go (TUG) test; the static balance, by means of the Functional Reach Test (FRT); and the muscle strength (MS) of the lower limbs, through the Short Physical Performance Battery (SPPB) test. The elderly were divided into: group HAS (hypertensive) and control group (nonhypertensive). The data was analyzed with use of the Graph Pad Prism Software, and unpaired Student's t-test was applied for identification of differences between the groups. Results: Of the 88 volunteers, 43 belong to the hypertensive group and 45 to the control group. For the 
FRT, group HAS had a mean functional reach measuring 15 ( \pm 5.60$)$ centimeters, while the control group had an average of 17 ( \pm 5.88$)$ centimeters. In the SPPB test, the mean MS for group HAS was 17.67 ( \pm 6.46$)$ seconds and, for the control group, it was 17.01 ( \pm 3.53$)$ seconds. The mean TUG for group HAS was 14.03 ( \pm 2.95$)$ seconds and, for the control group, it was 12.41 ( \pm 3.90$)$ seconds. There were associations between groups HAS and Control for FRT $(p=0.03)$ and SPPB $(p=0.02)$. Conclusion: Systemic arterial hypertension seems to interfere with static posture maintenance in the elderly.

Descriptors: Hypertension; Elderly; Postural Balance.

\section{RESUMEN}

Objetivo: Comparar la capacidad del mantenimiento de la postura estática y dinámica de ancianos hipertensos y no hipertensos en actividades funcionales. Métodos: Participaron de ese estudio individuos con edad mayor o igual a 60 años vinculados a una Unidad Básica de Salud (UBS) de un municipio pequeño del norte del Estado de Piauí. La capacidad del mantenimiento de la postura ha sido medida a través de tres pruebas. La movilidad funcional fue evaluada a través del test Timed Up and Go (TUG); el equilibrio estático con el Teste de Alcance Funcional (TAF); y la fuerza muscular (FM) de los miembros inferiores con el teste Short Physical Performance Battery (SPPB). Los ancianos se dividieron en: grupo HAS (hipertensos) y grupo Control (no hipertensos). Se analizaron los datos con el Software Graph Pad Prism utilizando la prueba t de student no pareado para identificar las diferencias entre los grupos. Resultados: De los 88 voluntarios, 43 son del grupo de hipertensos y 45 del grupo control. El grupo HAS tuvo la media de la mensuración del alcance funcional en el TAF de $15( \pm 5,60)$ centímetros; el grupo control tuvo una media de $17( \pm 5,88)$ centímetros. La media de la FM en el teste SPPB para el grupo HAS

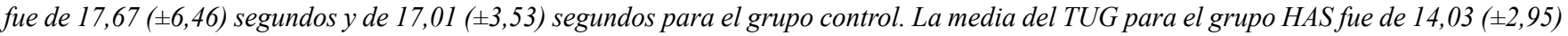
segundos y 12,41 ( $\pm 3,90)$ segundos para el grupo control. Hubo asociación entre los grupos HAS y control para los testes TAF ( $p=0,03) y$ $S P P B(p=0,02)$. Conclusión: La hipertensión arterial sistémica parece influenciar en la manutención de la postura estática de ancianos.

Descriptores: Hipertensión; Anciano; Balance Postural.

\section{INTRODUÇÃO}

A população idosa tem aumentado progressivamente nos últimos anos e, segundo a Organização Mundial da Saúde (OMS), o percentual de idosos será duplicado mundialmente até o ano de 2050 , passando de $11 \%$ para $22 \%{ }^{(1)}$. O mesmo comportamento tem sido observado no Brasil e de maneira ainda mais incisiva, devendo o número de idosos crescer de forma mais rápida que a média mundial. O percentual de idosos na população brasileira é estimado em $12,5 \%$, devendo alcançar os $30 \%$ até a metade do século $\mathrm{XXI}^{(2)}$.

O aumento na população de idosos deve-se à melhoria nas condições gerais de saúde, destacando-se a qualidade de vida, avanços na área da saúde, diminuição dos índices de natalidade e mortalidade ${ }^{(3,4)}$. Estes fatos proporcionaram mudanças no perfil de doenças recorrentes, repercutindo na atenção e promoção a saúde e políticas públicas ${ }^{(5)}$. A garantia da autonomia e fornecimento de suporte social ao indivíduo idoso devem ter especial atenção, surtindo efeitos positivos nas variadas formas de prestação de assistência ${ }^{(6)}$.

O processo natural de envelhecimento acarreta ao ser humano prejuízos funcionais que comprometem a capacidade de manutenção do equilíbrio corporal ${ }^{(7,8)}$. Essa repercussão é resultado da perda de massa muscular e diminuição das respostas adaptativas de equilíbrio e do processamento de informações no Sistema Nervoso Central (SNC) ${ }^{(9)}$. Todas essas alterações geram desequilíbrios, interferindo diretamente na capacidade de locomoção dos idosos ${ }^{(10)}$.

$\mathrm{O}$ processo de envelhecimento leva a uma progressiva diminuição da função de diversos sistemas, culminando em comprometimentos funcionais associados ao surgimento de doenças crônicas e degenerativas ${ }^{(2,11)}$. A Hipertensão Arterial Sistêmica (HAS) é uma das patologias mais comuns nessa faixa etária, sendo resultado da progressão da senescência, que gera redução da capacidade do sistema cardiovascular em estabelecer a homeostase ${ }^{(5,12)}$. A correlação entre HAS e alterações no equilíbrio já foi observada anteriormente, seja em idosos ${ }^{(13)}$, seja em jovens adultos ${ }^{(14)}$. Além desta propensão, característica dos idosos, há a influência dos hábitos de vida, que tendem a criar mecanismos fisiopatológicos que aumentam o risco de surgimento e de gravidade dessa morbidade ${ }^{(7,9,15)}$.

A inatividade física é um agravante na população geriátrica ${ }^{(16)}$, pois tende a acelerar o declínio morfofuncional dos sistemas nervoso e vestibular, implicando em inabilidade para estabelecer a manutenção postural estática ou dinâmica ${ }^{(17)}$. Dessa forma, as atividades funcionais cotidianas são comprometidas, gerando repercussões nos mais variados sistemas (musculoesquelético, cardiovascular e neurológico), surtindo efeitos danosos à saúde do idoso ${ }^{(6,10,12)}$. A partir dessas considerações, o objetivo deste estudo foi comparar a capacidade de manutenção postural estática e dinâmica em atividades funcionais entre idosos hipertensos e não hipertensos. 


\section{MÉTODOS}

Tratou-se de uma pesquisa quantitativa do tipo transversal, de caráter exploratório e descritivo. A pesquisa foi desenvolvida em uma UBS de um município de pequeno porte no norte do Estado do Piauí no período de novembro de 2014 a janeiro de 2015. O município apresenta uma população de 10,4\% de pessoas com 60 anos ou mais de idade ${ }^{(2)}$ e a UBS escolhida para o estudo assiste 163 idosos. Para a escolha da UBS foi realizado um sorteio dentre as 6 unidades que se encontram na zona urbana do município. Um total de 95 idosos compareceram a UBS para serem voluntários no estudo ${ }^{(18)}$.

Incluíram-se na pesquisa idosos cadastrados nessa determinada UBS, que concordaram em participar do estudo e confirmaram o aceite de participação assinando um Termo de Consentimento Livre e Esclarecido (TCLE); os idosos precisavam apresentar autonomia física e mental. Cada participante foi orientado quanto aos estágios da pesquisa e questões como anonimato e desistência.

Foram excluídos os voluntários que não completaram a avaliação por possuírem comprometimento significativo da autonomia física e mental, por alterações cognitivas e motoras que pudessem repercutir na execução dos testes propostos.

Todos os dados de anamnese deste estudo foram obtidos pelos avaliadores em parceria com Agentes Comunitários de Saúde (ACS) da UBS.

Realizou-se uma entrevista com os participantes que apresentaram o perfil da pesquisa. Nessa etapa, os idosos responderam a um questionário que possibilitou a coleta de informações como idade, gênero, escolaridade, renda, situação conjugal, dados antropométricos e comorbidades, e nível de atividade física (auto percepção do paciente).

Os requisitos cinéticos funcionais para manutenção postural estática e dinâmica dos membros inferiores dos idosos foram avaliados. Dessa forma, a manutenção da postura corporal estática foi simulada através de um teste do equilíbrio estático e a manutenção da postura corporal dinâmica foi simulada através de testes que avaliam a força muscular dos membros inferiores e a mobilidade funcional. Os testes foram aplicados por um avaliador previamente treinado e sem conhecimento precedente do diagnóstico de HAS no voluntário.

O equilíbrio estático foi estimado por meio do Teste de Alcance Funcional (TAF), em que o idoso avaliado encontravase em posição ortostática, membros inferiores levemente abduzidos, coluna ereta, com a visão direcionada para o horizonte, ombro do membro superior direito (MSD) fletido a $90^{\circ}$, cotovelo e punho estendido em toda sua amplitude. O hemicorpo direito sendo mantido próximo à parede, com solicitação ao avaliado que deslocasse o seu MSD anteriormente, o máximo possível. A excursão do braço, tendo como marcação de partida a extremidade do $3^{\circ}$ metacarpo, desde o início até o fim do teste foi mensurada utilizando uma fita métrica fixada na parede no sentido horizontal ao lado do voluntário, na altura do acrômio. Deslocamentos menores que $15 \mathrm{~cm}$ indicam fragilidade e risco ao evento queda ${ }^{(19)}$.

A força muscular (FM) dos membros inferiores (MMII) foi avaliada pelo teste Short Physical Performance Battery (SPPB), realizado por meio do sentar-se e levantar-se da cadeira 5 vezes consecutivas, sem utilizar os membros superiores, sendo cronometrado o tempo de execução da manobra. A pontuação máxima, de 4 pontos foi atribuída para um tempo de 11,19 segundos ou menos de realização do teste; para um tempo de teste de 11,20 a 13,69 segundos, 3 pontos; para 13,70 a 16,69 segundos de tempo, 2 pontos, e para 16,70 segundos ou mais, 1 ponto. Caso o sujeito não consiga levantar-se cinco vezes da cadeira ou completar o teste em tempo maior que 60 segundos, não é atribuída nenhuma pontuação ${ }^{(20,21)}$.

A mobilidade funcional foi ponderada através do teste Timed Up and Go (TUG), que mensura em segundos o tempo gasto por um indivíduo para levantar-se de uma cadeira, deambular $3 \mathrm{~m}$, dar a volta, caminhar de volta à cadeira e sentar-se novamente ${ }^{(22)}$. Para fazer o teste, foi utilizado um cronômetro digital marca Kenko (modelo KK-2808 - China), uma cadeira com altura de $46 \mathrm{~cm}$ com apoio para os braços. O voluntário foi posicionado em sedestação, com as costas apoiadas e os pés paralelos ao chão, e recebia informações prévias sobre a execução do teste. O TUG teve início com o comando verbal do avaliador, então imediatamente o participante erguia-se da cadeira simultâneo ao começo da contagem do tempo de execução do teste. O tempo gasto para realizar o teste está relacionado ao risco do evento queda, considerando-se a que a execução em menos de 20 segundos indica um pequeno risco de queda; entre 20 e 30 segundos, um risco moderado; e um grande risco de queda se demorar mais que 30 segundos $^{(23)}$.

Após a aplicação dos testes, os resultados gerados foram divididos em dois grupos de acordo com o diagnóstico de HAS, que foi determinado pelo médico responsável por cada participante, mas sendo essa uma atividade independente deste estudo. As informações foram relatadas pelos idosos e averiguadas com os exames trazidos.

O teste $t$ student não pareado foi aplicado para identificar diferenças entre os grupos experimentais em cada variável analisada. O nível de significância adotado foi de $5 \%(\mathrm{p}<0,05)$. Todos os dados foram analisados através do Software Graph Pad Prism (versão seis) para Windows.

A pesquisa seguiu os princípios da Resolução nº. 466/2012 do Conselho Nacional de Saúde (CNS) e os procedimentos relacionados à coleta e análise de dados receberam aprovação da Comissão de Ética em Pesquisa do Centro de Ensino Unificado de Teresina (CEUT), sob número de protocolo 6597/2014. 


\section{RESULTADOS}

Dos 95 idosos inclusos inicialmente na amostra, um total de 88 compareceram no momento da avaliação. Destes, 43 idosos integraram o grupo HAS e 45 o grupo controle. As caracterizações antropométricas e sociodemográficas dos grupos estão descritas na tabela I.

Tabela I - Caracterização antropométrica e sócio-demográfica de idosos assistidos em uma Unidade Básica de Saúde de uma cidade do norte do Piauí. 2014-2015.

\begin{tabular}{|c|c|c|c|c|c|c|c|}
\hline \multirow{2}{*}{ Característica } & & \multicolumn{2}{|c|}{ Grupo HAS } & \multicolumn{2}{|c|}{ Grupo controle } & \multicolumn{2}{|c|}{ Total } \\
\hline & & $\mathbf{n}$ & $\%$ & $\mathbf{n}$ & $\%$ & $\mathbf{n}$ & $\%$ \\
\hline \multirow{4}{*}{ Sexo } & Masculino & 18 & 41,8 & 19 & 42,3 & 37 & 42,1 \\
\hline & Feminino & 25 & 58,2 & 26 & 57,7 & 51 & 57,9 \\
\hline & $60-64$ & 11 & 25,6 & 12 & 26,7 & 23 & 26,1 \\
\hline & $65-69$ & 7 & 16,3 & 18 & 40,0 & 25 & 55,5 \\
\hline \multirow[t]{4}{*}{ Idade } & $70-74$ & 16 & 37,2 & 8 & 17,7 & 24 & 53,4 \\
\hline & $75-79$ & 3 & 6,7 & 5 & 8,8 & 8 & 9,0 \\
\hline & $>80$ & 6 & 13,3 & 2 & 4,4 & 8 & 9,0 \\
\hline & Abaixo do peso & 0 & 0 & 5 & 11,0 & 5 & 56,8 \\
\hline \multirow{4}{*}{ IMC } & Peso normal & 14 & 32,6 & 24 & 53,3 & 38 & 43,1 \\
\hline & Sobrepeso & 22 & 51,1 & 15 & 33,3 & 37 & 42 \\
\hline & Obesidade grau 1 & 5 & 11,6 & 2 & 4,4 & 7 & 7,9 \\
\hline & Casado & 25 & 58,1 & 31 & 68,88 & 56 & 63,6 \\
\hline \multirow{4}{*}{ Estado conjugal } & Solteiro & 2 & 4,6 & 0 & 0 & 2 & 2,3 \\
\hline & Divorciado & 1 & 2,3 & 6 & 13,3 & 7 & 7,9 \\
\hline & Viúvo & 15 & 34,8 & 8 & 17,7 & 23 & 26,1 \\
\hline & Analfabeto & 29 & 67,4 & 30 & 66,7 & 59 & 67,0 \\
\hline \multirow[t]{3}{*}{ Escolaridade } & Ens. Fund. Incompleto & 14 & 32,6 & 14 & 31,1 & 28 & 31,8 \\
\hline & Ens. Fund. Completo & 0 & 0 & 1 & 2,2 & 1 & 1,1 \\
\hline & $<1$ salário & 2 & 4,6 & 0 & 0 & 2 & 2,3 \\
\hline \multirow[t]{2}{*}{ Renda familiar } & 1 salário & 39 & 90,8 & 44 & 97,7 & 83 & 94,3 \\
\hline & $>1$ salário & 2 & 4,6 & 1 & 2,3 & 3 & 3,4 \\
\hline
\end{tabular}

HAS: Hipertensão Arterial Sistêmica; IMC: Índice de Massa Corporal. Ens. Fund.: Ensino Fundamental.

No grupo HAS, o histórico de quedas esteve presente em 48,83\% (n=21) dos avaliados, o diagnóstico de Diabetes Mellitus (DM) prevaleceu entre 27,9\% (n=12) sujeitos e o sedentarismo (não realização de atividade física por no mínimo 30 minutos pelo menos três vezes por semana) foi observado em $67,4 \%(\mathrm{n}=29)$. No grupo controle, o histórico de quedas foi verificado em 44,44\% (n=20) dos avaliados, não se observou diagnóstico de DM e a presença de sedentarismo alcançou 55,5\% (n=25).

A análise estatística revelou que a houve diferença entre os grupos HAS e controle para os testes TAF e FM, respectivamente $\mathrm{p}=0,0399$ e $\mathrm{p}=0,0205$. No entanto, a comparação entre grupos por meio do TUG não revelou diferença entre os grupos $(\mathrm{p}=0,1749)$.

As figuras 1, 2 e 3 mostram as comparações entre ambos para os testes TAF, FM e TUG.

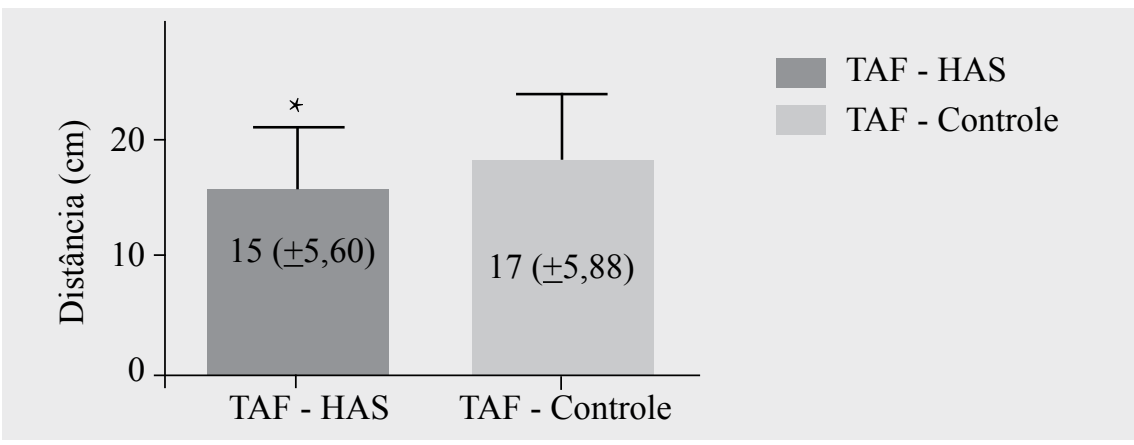

Figura 1 - Comparação do equilíbrio estático entre os grupos de idosos hipertensos (HAS) e não hipertensos (controle) assistidos em uma Unidade Básica de Saúde de uma cidade do norte do Piauí. 2014-2015.

HAS: Hipertensão Arterial Sistêmica; Teste de Alcance Funcional (TAF). *p<0,05. 


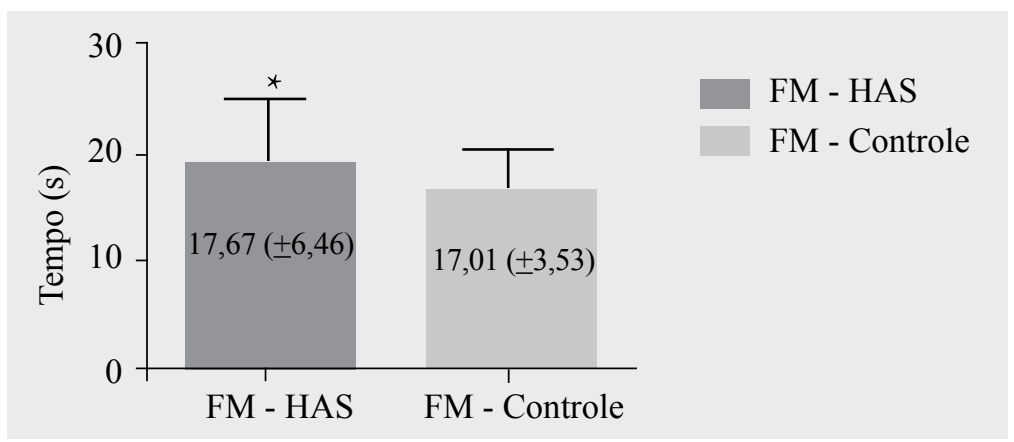

Figura 2 - Comparação da força muscular (FM) entre os grupos de idosos hipertensos (HAS) e não hipertensos (controle) assistidos em uma Unidade Básica de Saúde de uma cidade do norte do Piauí. 2014-2015.

HAS: Hipertensão Arterial Sistêmica; FM: Força Muscular. ${ }^{*} \mathrm{p}<0,05$.

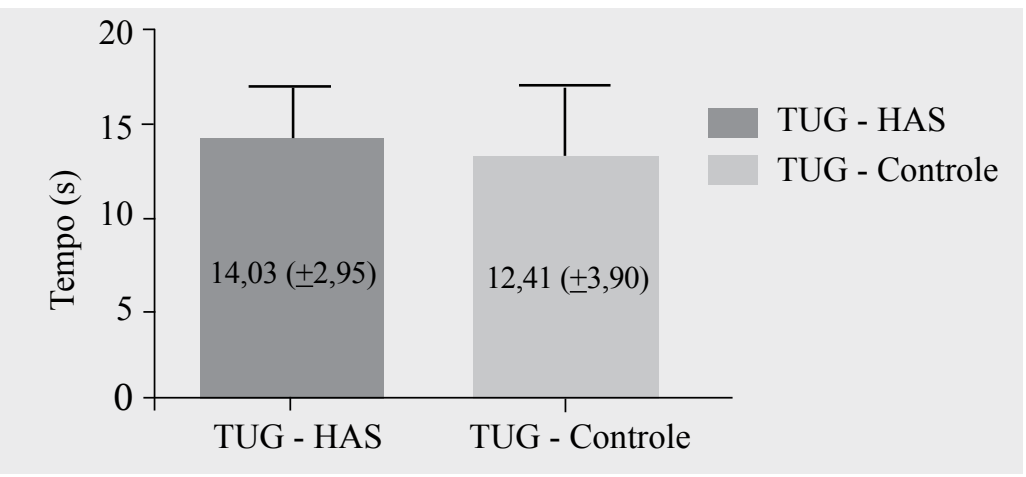

Figura 3 - Comparação do equilíbrio estático entre os grupos de idosos hipertensos (HAS) e não hipertensos (controle) assistidos em uma Unidade Básica de Saúde de uma cidade do norte do Piauí. 2014-2015.

HAS: Hipertensão Arterial Sistêmica; TUG: Timed Up and Go. ${ }^{*} \mathrm{p}<0,05$.

\section{DISCUSSÃO}

Os resultados encontrados mostraram que os idosos não hipertensos tiveram melhor desempenho no equilíbrio estático e no teste de força muscular. Essa temática exige uma contextualização de fatores que são determinantes na repercussão da hipertensão em idosos, como adesão ao tratamento, hábitos de vida saudáveis e a presença de outras morbidades que comumente acometem os indivíduos hipertensos ${ }^{(24,25)}$.

As características dos grupos foram semelhantes em vários fatores sociodemográficos. A maioria dos idosos participantes deste estudo tiveram predominância nas seguintes características: casados, residindo com o cônjuge, analfabetos, sustentandose mensalmente com um salário. Algumas dessas características são relevantes para a adesão de hábitos de vida saudáveis, como o analfabetismo, que dificulta o acesso à informação e compreensão dos aspectos preventivos e terapêuticos das doenças crônicas ${ }^{(26)}$. A baixa renda limita a possibilidade de execução dessas medidas ${ }^{(27)}$.

A mobilidade funcional foi avaliada através do TUG. Este instrumento é útil para identificar problemas que afetam o equilíbrio dos idosos nas suas atividades de vida diária de forma simples, eficaz, rápida e sem gastos excessivos ${ }^{(28)}$. No presente estudo os indivíduos hipertensos tiveram a mesma agilidade e equilíbrio para executar o teste que o controle, este resultado diverge com os achados de uma revisão integrativa que incluiu publicações do ano de 2007 a $2014^{(26)}$, esta revisão sugere que as comorbidades que acometem os indivíduos hipertensos ocasionam efeitos deletérios em seus portadores potencializando o comprometimento funcional.

A atividade física é recomendada como forma de prevenção e tratamento não farmacológico da hipertensão arterial ${ }^{(29,30)}$. Apesar de haver esse incentivo à adesão aos programas de atividade física, a maioria dos idosos foram considerados sedentários, o que mostra uma carência de estratégias de intervenção junto à população idosa acerca dos prejuízos advindos do sedentarismo ${ }^{(31)}$.

Para manutenção postural estática e dinâmica se faz necessária ação muscular, viabilizando a sustentação e os ajustes necessários para manter o equilíbrio. Neste estudo, optou-se por utilizar o teste Short Physical Performance Battery (SPPB) para avaliar a FM dos MMII, pois a sua execução requer a habilidade do músculo exercer grande quantidade de força em velocidade $^{(21)}$. Sentar e levantar é um exemplo clássico de realização de atividade básica e instrumental de vida diária que necessita da potência muscular(20,32). 
Os idosos hipertensos tiveram pior desempenho na realização do teste de FM, demorando mais tempo para finalizá-lo. A flexibilidade muscular pode interferir na capacidade da contração muscular gerar força, fator que pode ter contribuído para um pior desempenho dos idosos com hipertensão. Essa hipótese é fundamentada por alguns autores que sugerem ${ }^{(33)}$ que idosos hipertensos apresentam menor flexibilidade nos músculos dos MMII, sobretudo nos músculos sóleo e gastrocnêmio. Sugere-se que essa característica também repercutiu nos resultados do TAF. O grupo HAS teve um menor alcance anterior nesse teste, podendo inferir menor equilíbrio, mas também limitação biomecânica por encurtamento das cadeias musculares de membros inferiores ${ }^{(6,11)}$.

Nesse contexto, tem-se considerado que as perdas musculares e funcionais se aceleram depois dos 70 anos, quando a atrofia e fraqueza muscular parecem ser de evolução mais rápida ${ }^{(34)}$. Considerando a média absoluta das idades dos participantes o grupo HAS teve uma faixa etária discretamente maior. Esta característica pode otimizar a repercussão funcional deletéria do envelhecimento, entretanto, no presente estudo não houve relevância estatística que pudesse determinar tal influência.

O DM compromete o sistema locomotor, o controle postural e o equilíbrio, reduzindo as respostas adaptativas e contribuindo

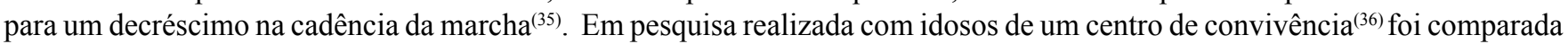
a mobilidade funcional de idosos diabéticos e não diabéticos e conclui-se uma menor mobilidade funcional associada ao diagnóstico de DM. Achado semelhante a outros autores que abordaram a mesma temática, inclusive com repercussão na força muscular ${ }^{(10,11)}$. A diabetes foi identificada apenas no grupo de hipertensos, com um percentual de $27,9 \%$. A influência dessa comorbidade não foi evidenciada nos resultados da mobilidade funcional, porém, pode ter repercutido na potência muscular e equilíbrio estático.

Para realizar atividades funcionais exercendo controle corporal estático e dinâmico, faz-se necessário aptidão nos requisitos cinéticos funcionais, como força muscular, equilíbrio estático e dinâmico ${ }^{(7,19,37)}$. Os testes realizados neste estudo buscaram a simulação dessas variáveis. Somente o TUG não diferiu estatisticamente entre os grupos, apesar de indivíduos hipertensos terem predisposição a alterações que podem prejudicar o desempenho neste teste ${ }^{(28)}$. A similaridade com relação à mobilidade funcional pode ser atribuída ao incentivo de exercício aeróbicos, como caminhadas. No entanto, novas modalidades de exercício devem ser implementadas visando estimular a potência muscular e a manutenção do equilíbrio ${ }^{(38)}$.

Deve-se buscar estratégias que visem manutenção da independência funcional na atenção à saúde da população geriátrica ${ }^{(5)}$. Instrumentos de avaliação devem ser utilizados para identificar precocemente as repercussões do envelhecimento a fim de elaborar programas de intervenção que sejam objetivos às principais necessidades dos idosos ${ }^{(32,39)}$. Umas das limitações deste estudo foi a utilização de recursos restritos para investigação dos sistemas relacionados ao controle postural. Entretanto, esses recursos mais detalhistas de avaliação são comuns apenas em centros de atenção de média a alta complexidade, pois são bastante onerosos para uso na atenção básica. A contribuição deste estudo para a comunidade científica e populacional é o uso de instrumentos de baixo custo, fácil aplicabilidade e que possuem sua fidedignidade amplamente discutida na literatura, podendo contribuir com efetividade na atenção básica.

\section{CONCLUSÃO}

Os idosos hipertensos avaliados apresentaram menor funcionalidade no equilíbrio estático e força muscular, assim, a hipertensão arterial sistêmica parece interferir na manutenção postural estática em idosos.

\section{REFERÊNCIAS}

1. World Health Organization. Health of the elderly [acesso em 2014 Jun 20]. Disponível em: http://www.emro.who.int/ entity/elderly-health/index.html

2. Ministério da Saúde (BR). Saúde da pessoa idosa [acesso em 2016 Jan 20]. Disponível em: http://portalsaude.saude.gov. br/index.php/oministerio/principal/secretarias/sas/daet/saude-da-pessoa-idosa

3. Küchemann BA. Envelhecimento populacional, cuidado e cidadania: velhos dilemas e novos desafios. Soc Estado. 2012;27(1):165-80.

4. Paula JM, Sawada NO, Nicolussi AC, Andrade CTAE, Andrade V. Qualidade de vida em idosos com mobilidade física prejudicada. Rev Rene. 2013;14(6):1224-31.

5. Gomes DBC, Bezerra EMA, Silva JCA, Costa TPS, Ribeiro MDA, Campelo GO. Avaliação da qualidade de vida em idosos hipertensos atendidos em clínica de fisioterapia. Sanare (Sobral, Online). 2015 [acesso em 2016 Jan 20];14(1):33-7. Disponível em: https://sanare.emnuvens.com.br/sanare/article/view/605

6. Allendorf DB, Schopf PP, Gonçalves BC, Closs VE, Gottlieb MGV. Idosos praticantes de treinamento resistido apresentam melhor mobilidade do que idosos fisicamente ativos não praticantes. R Bras Ciênc Mov. 2016;24(1):134-44. 
7. Silva EKR, Macêdo LC. Realidade virtual no treinamento do equilíbrio em idosos: um estudo de revisão. Rev Pesquisa Fisioterapia. 2014;4(2):137-43.

8. Dourado CS, Santos EL, Romero JR, Alcaide AR, Silva AS. Efeitos do exercício resistido sobre a força muscular de idosos: uma revisão de literatura. Rev Inspirar. 2015;7(2):11-5.

9. Almeida ST, Soldera CLC, Carli GA, Gomes I, Resende TL. Análise de fatores extrínsecos intrínsecos que predispõem a quedas em idosos. Rev Assoc Med Bras. 2012;58(4):427-33.

10. Costa AGS, Araujo TL, Oliveira ARS, Morais HCC, Silva VM, Lopes MVO. Fatores de risco para quedas em idosos. Rev Rene. 2013;14(4):821-8.

11. Silva JR, Camargo RCT, Nunes MM, Camargo TT, Faria CRS, Abreu LC. Análise da alteração do equilíbrio, marcha e o risco de quedas em idosos praticantes de um programa de fisioterapia. Rev e-ciência. 2014;2(2):20-3.

12. Moroz MB, Kluthcovsky ACGC, Schafranski MD. Controle da pressão arterial em idosas hipertensas em uma unidade de saúde da família e fatores associados. Cad Saúde Colet (Rio J). 2016;24(1):111-7.

13. Steter AM, Coutinho RMC, Fernandes ACP, Costa LFV. Importância da avaliação do equilíbrio e da marcha do idoso. J Health Sci Inst. 2014;32(1):43-7.

14. Cruz IBM, Barreto DCM, Fronza AB, Jung IVC, Krewer CC, Rocha MIUM, et al. Dinamic balance, lifestyle and emotional states in Young adults. Braz J Otorhinolaryngol. 2010;76(3):392-8.

15. Silva LOL, Dias CA, Rodrigues SM, Soares MM, Oliveira MA, Machado CJ. Hipertensão arterial sistêmica: representações sociais de idosos sobre a doença e seu tratamento. Cad Saúde Colet (Rio J). 2013;21(2):121-8.

16. Massa KHC, Guimarães VV, César CLG, Barros MBA, Carandina L, Goldbaum M, et al. Atividade física e hipertensão em idosos no Município de São Paulo. Pelotas/RS. Rev Bras Ativ Fis Saúde. 2012;17(1):7-13.

17. Macedo C, Gazzola JM, Caovilla HH, Ricci NA, Doná F, Ganança FF. Posturografia em idosos com distúrbios vestibulares e quedas. ABCS Health Sci. 2013;38(1):17-24.

18. Secretaria Municipal de Saúde de Cocal/Piauí. Informações adquiridas do sistema HIPERDIA do município de Cocal-PI. Cocal; 2014

19. Souza LA, Fernandes AB, Patrizzi LJ, Walsh IAP, Shimano SGN. Efeitos de um treino multissensorial supervisionado por seis semanas no equilíbrio e na qualidade de vida de idosos. Medicina (Ribeirão Preto). 2016;49(3):223-31.

20. Miranda LV, Silva GCB, Meneses YPSF, Cortez ACL, Araújo DG, Gayoso JCA Neto. Efeitos de 9 semanas de treinamento funcional sobre índices de aptidão muscular de idosas. Rev Bras Prescrição Fisiol Exercício. 2016;10(59):386-94.

21. Pires MCO. Aplicabilidade da Short Physical Performance Battery na avaliação funcional de indivíduos com doença arterial obstrutiva periférica [dissertação]. Belo Horizonte: Universidade Federal de Minas Gerais; 2015.

22. Gonçalves TV, Pereira JGC, Castro HO, Leite RD, Pires FO. Influência aguda do alongamento prévio no desempenho do teste timed up and go (TUG) em idosas. Rev Bras Prescrição Fisiol Exercício. 2015;9(55):555-61.

23. Bretan O, Silva JE Júnior, Ribeiro OR, Corrente JE. Risk of falling among elderly persons living in the community: assessment by the timed up and go test. Braz J Otorhinolaryngol. 2013;79(1):18-21.

24. Silveira J, Scherer F, Deitos A, Bosco SMD. Fatores associados à hipertensão arterial sistêmica e ao estado nutricional de hipertensos inscritos no programa hiperdia. Cad Saúde Colet (Rio J). 2013;21(2):129-34.

25. Andrade AO, Aguiar MIF, Almeida PC, Chaves ES, Araujo NVSS, Freitas JB Neto. Prevalência da hipertensão arterial e fatores associados em idosos. Rev Bras Promoç Saúde. 2014;27(3):303-11.

26. Nunes LCSM, Santos CAA, Serra MAAO. Fatores de risco e cuidados de enfermagem ao idoso hipertenso: revisão integrativa. Sanare (Sobral). 2014;13(2):103-9.

27. Andrade JMO, Rios LR, Teixeira LS, Vieira FS, Mendes DC, Vieira MA, et al. Influência de fatores socioeconômicos na qualidade de vida de idosos hipertensos. Ciênc Saúde Coletiva. 2014;19(8):3497-504.

28. Campos MPS, Vianna LG, Campos AR. Os testes de equilíbrio alcance funcional e "Timed Up and Go" e o risco de quedas em idosos. Rev Kairós. 2013;16(4):125-38.

29. Kosarger Larser M, Matchkov VV. Hypertension and physical exercise: the role of oxidative stress. Medicina (Kaunas). 2016;52(1):19-27. 
30. Ponte MKC, Cunha FMAM. Nível de atividade física na população idosa e seus benefícios: uma revisão integrativa. Sanare (Sobral). 2013;12(1):71-7.

31. Nascimento SM, Pinheiro RS. Participação no programa de atividade física de idosos residentes em uma casa gerontológica no Rio de Janeiro. Cad Saúde Colet (Rio J). 2013;21(1):46-52.

32. Picorelli AMA, Pereira DS, Felício DC, Gomes DA, Dias RC, Pereira LCM. Adesão de idosas a um programa de exercícios domiciliares pós-treinamento ambulatorial. Fisioter Pesqui. 2015;22(3):291-308.

33. Garcia PA, Dias JMD, Dias RC, Santos P, Zampa CC. Estudo da relação entre função muscular, mobilidade functional e nível de atividade física em idosos comunitários. Rev Bras Fisioter. 2011;15(1):15-22.

34. Cadore EL, Rodríguez-Mañas L, Sinclair A, Izquierdo M. Effects of diferente exercise interventions on risk of falls, gait ability and balance in physically frail older adults: a systematic review. Rejuvenation Res. 2013;16(2):105-14.

35. Oliveira PP, Fachin SM, Tozatti J, Ferreira MC, Marinheiro LPF. Análise comparativa do risco de quedas entre pacientes com e sem diabetes mellitus tipo 2. Rev Assoc Med Bras 2012;58(2):234-9

36. Silva JCA, Silva LN, Gomes DBC, Linhares KP, Couto LC, Moreira AKF. Análise comparativa do risco queda em idosas diabéticas e não diabéticas de Parnaíba-PI. Rev Inspirar. 2013;5(6):11-15

37. Santos FPV, Borges LL, Menezes RL. Correlação entre três instrumentos de avaliação para risco de queda em idosos. Fisioter Mov. 2013;26(4):883-94.

38. Ambrose AF, Paul G, Hausdorff JM. Risk factors for falls among older adults: a review of the literature. Maturitas. 2013;75(1):51-61.

39. Gomes GAO, Cintra FA, Diogo MJD, Neri AL, Guariento ME, Sousa MLR. Comparação entre idosos que sofreram quedas segundo desempenho físico e número de ocorrências. Rev Bras Fisioter. 2009;13(5):430-7.

\section{Endereço para correspondência:}

Jefferson Carlos Araujo Silva

Universidade Federal do Maranhão - UFMA

Hospital Universitário Presidente Dutra

Rua Barão de Itapary, 227

Bairro: Centro

CEP: 65.020-070 - São Luís - MA - Brasil

E-mail: jeffcasilva@gmail.com 